\title{
Crystallization dynamics on curved surfaces
}

\author{
Nicolás A. García, ${ }^{1}$ Richard A. Register, ${ }^{2}$ Daniel A. Vega, ${ }^{1, *}$ and Leopoldo R. Gómez ${ }^{1, \dagger}$ \\ ${ }^{1}$ Department of Physics, Universidad Nacional del Sur - IFISUR - CONICET, 8000 Bahía Blanca, Argentina \\ ${ }^{2}$ Department of Chemical and Biological Engineering, Princeton University, Princeton, New Jersey 08544, USA
}

(Received 11 March 2013; revised manuscript received 8 July 2013; published 31 July 2013)

\begin{abstract}
We study the evolution from a liquid to a crystal phase in two-dimensional curved space. At early times, while crystal seeds grow preferentially in regions of low curvature, the lattice frustration produced in regions with high curvature is rapidly relaxed through isolated defects. Further relaxation involves a mechanism of crystal growth and defect annihilation where regions with high curvature act as sinks for the diffusion of domain walls. The pinning of grain boundaries at regions of low curvature leads to the formation of a metastable structure of defects, characterized by asymptotically slow dynamics of ordering and activation energies dictated by the largest curvatures of the system. These glassylike ordering dynamics may completely inhibit the appearance of the ground-state structures.
\end{abstract}

DOI: 10.1103/PhysRevE.88.012306

PACS number(s): 64.70.dg, 61.30.Hn, 61.72.Cc

\section{INTRODUCTION}

The evolution from a liquid to a crystal structure is one of the oldest problems in condensed matter, with wide interest in both basic science and technology [1]. In three-dimensional space the mechanism of crystallization is still a matter of ongoing research. Since in general the seeds for nucleation do not have the same symmetry as the equilibrium structure, the early process of crystallization is a competition among different intermediate metastable states [1-4]. On the other hand, in two-dimensional flat space the physics behind crystallization is much simpler because there is no frustration to nucleating the equilibrium structure, such that there is no need for intermediate precursors [5]. However, two-dimensional (2D) crystals deposited on curved surfaces come back to complexity. Here the underlying curvature locally frustrates the formation of the crystal lattice, modifying both equilibrium structures [6-16] and their dynamical properties $[17,18]$.

Curved crystalline structures are ubiquitous in nature. For example, they can be found in viral capsids, insect eyes, pollen grains, and radiolaria. During the past decade these crystals have attracted the interest of different communities because of the richness associated with the coupling between geometry, structure, and functionality. Recently, curved crystals have been obtained in a controlled fashion by the use of colloidal matter $[9,14,17,18]$. Other self-assembled systems with great potential to develop such structures are block copolymers and liquid crystals [19-26]. In curved crystals, defects can be a feature of the fundamental (equilibrium) state. Depending on the substrate's topology and curvature, defects can be required to reduce lattice distortions and to satisfy topological constraints. Thus, from a condensed-matter perspective, the presence of curvature in ordered phases appears as an opportunity for accurate control of the density and location of topological defects [27,28].

Although theoretical and experimental work has led to a substantial advance in the knowledge of equilibrium structures and features, the out-of-equilibrium dynamics leading to the

\footnotetext{
*dvega@uns.edu.ar

†'lgomez@uns.edu.ar
}

formation of curved crystals, highly relevant for technological applications like defect functionalization engineering or soft lithography, remain almost unexplored.

In this work we use a free-energy functional that includes competing interactions to describe the large-scale dissipative dynamics of crystallization in systems residing on curved backgrounds. This simple model captures the essential features of crystallization over diffusive time scales and provides a clear picture about the complex coupling between geometry and crystallinity. We focus on the long-time dynamics of the system which is governed by the formation, interaction, and annihilation of topological defects.

\section{MODEL AND ANALYSIS}

The process of crystallization in curved space can be described by the following free-energy functional:

$$
\begin{aligned}
F= & \int d^{2} r \sqrt{g}\left[W(\psi)+\frac{D}{2} g^{\alpha \beta} \partial_{\alpha} \psi(\mathbf{r}) \partial_{\beta} \psi(\mathbf{r})\right] \\
& -\frac{b}{2} \iint d^{2} r d^{2} r^{\prime} \sqrt{g} \sqrt{g^{\prime}} G\left(\mathbf{r}-\mathbf{r}^{\prime}\right) \psi(\mathbf{r}) \psi\left(\mathbf{r}^{\prime}\right) .
\end{aligned}
$$

Here $\psi$ is an order parameter related to fluctuations of the density from the average, $W(\psi)=-\gamma \psi^{2}+v \psi^{3}+u \psi^{4}$ is a double-well potential that below the critical temperature presents two minima, $D$ is a penalty to form interfaces, $\sqrt{g}$ is the determinant of the metric $g_{\alpha \beta}$ of the curved substrate, and $G\left(\mathbf{r}-\mathbf{r}^{\prime}\right)$ is a Green's function which takes into account long-range interactions leading to the formation of a local hexagonal structure. Equation (1) is the covariant generalization of models widely used to study properties of systems with competing interactions with direct applications to a large collection of condensed systems: block copolymers, crystals, magnetic multilayer compounds, Rayleigh-Bénard convection patterns, and doped Mott insulators [29,30], among others. Close to the critical line, Eq. (1) reduces to the Landau-Brazovsky free-energy functional which has been employed for years in the understanding of the liquid to crystal transition in Euclidean space.

The dynamics leading to crystallization can be analyzed by considering the temporal evolution of a liquid phase quenched 


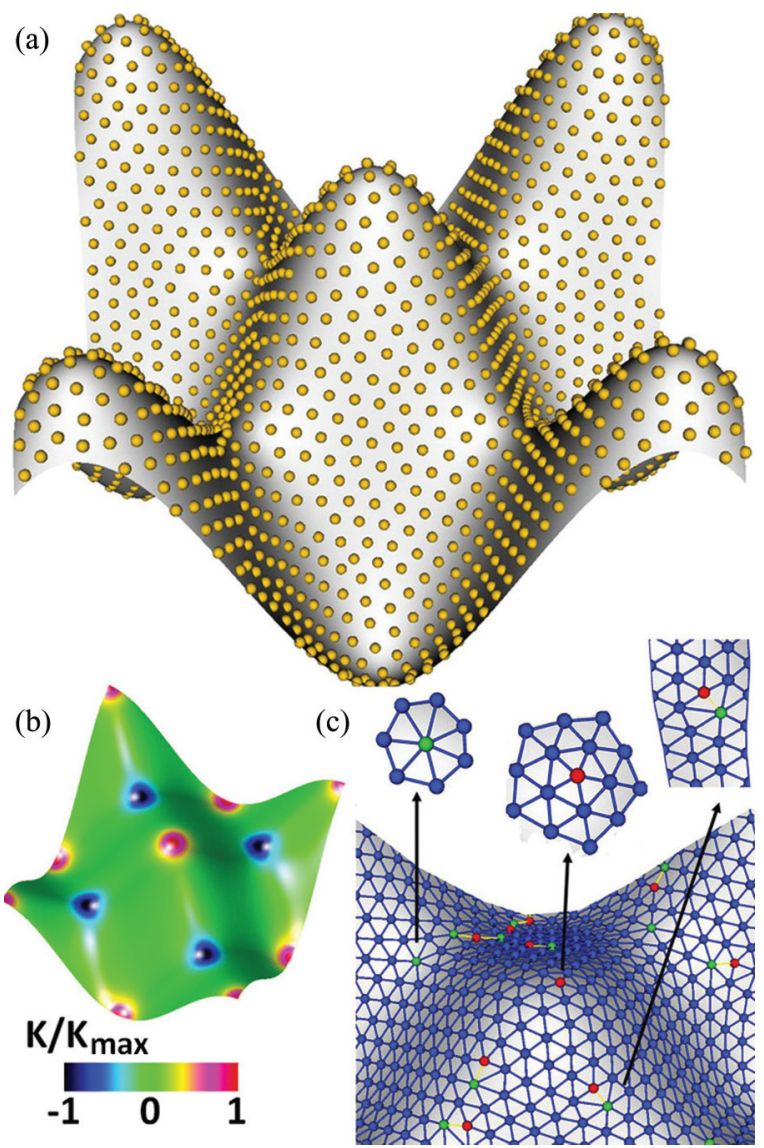

FIG. 1. (Color online) (a) Relaxed configuration of a hexagonal crystal lying on a sinusoidal substrate. (b) Distribution of Gaussian curvature. Here $K_{\max }$ is the maximum curvature. (c) Delaunay triangulations are used to identify the topological defects like negative (left) and positive (center) disclinations, and dislocations (right).

below the critical temperature, through the evolution equation:

$$
\frac{\partial \psi}{\partial t}=M \nabla_{\mathrm{LB}}^{2}\left\{\frac{\delta F}{\delta \psi}\right\} .
$$

Here $M$ is a phenomenological mobility coefficient and $\nabla_{\mathrm{LB}}^{2}$ is the Laplace-Beltrami operator which reduces to the classical Laplacian in flat geometries (see Appendix A for details on the evolution equation and numerical methods).

In this work we focus on the crystallization of hexagonal systems residing on sinusoidal substrates of different curvatures [Fig. 1(a)], characterized by an amplitude $A$ and a wavelength $L$ larger than the lattice constant in the crystal. In general, for every regular point $P$ on the surface there are two tangent circles with maximal and minimal radii of curvature $R_{1}$ and $R_{2}$, respectively [31]. The Gaussian curvature $K$ at $P$ is then defined as $K=\kappa_{1} \kappa_{2}$, where $\kappa_{i}=1 / R_{i}, i=1,2$ are the principal curvatures. The Gaussian curvature represents the intrinsic curvature of the surface. In the geometry studied here it takes on negative (positive) values in the neighborhood of the saddles (crests or valleys). Regions of zero Gaussian curvature correspond to points resembling the flat Euclidean plane. Note that for these surfaces the curvature is symmetrically distributed [see Fig. 1(b)], such that the integrated curvature is zero. Thus this system is topologically equivalent to the plane, such that no defects are geometrically required to form the crystal lattice; defect structures, if any, must arise only from energetic considerations.

Upon crystallization, the positions of the particles can be determined through the local maxima in the order parameter function $\psi(\mathbf{r})$. Once the particles' positions are known, the temporal evolution of the coordination number, particles' first neighbors, degree of crystallinity, pair-correlation function, and topological defects can be analyzed through Delaunay triangulations (see Appendix B for supplementary information about the method used to obtain Delaunay triangulations on curved surfaces).

The most common defects of a hexagonal flat crystal are given by particles whose coordination number differs from 6 [Fig.1(c)] [1]. Five- and seven-coordinated particles, named positive and negative disclinations, respectively, are deeply involved in the high-temperature behavior of $2 \mathrm{D}$ crystals and also unavoidably created during a symmetry-breaking liquid-to-crystal transition. In flat systems these topological defects are highly energetic because they produce large distortions in the crystal lattice. Consequently, in flat crystals disclinations are not found isolated but are arranged in dipoles, known as dislocations [Fig. 1(c)]. On the contrary, on curved substrates the disclinations can help to screen out the geometric frustration induced by the substrate's geometry, reducing the elastic distortions generated by the curvature [6-16]. Thus, in curved crystals, the disclinations are not necessarily defects as they can belong to the equilibrium state of the crystal.

\section{RESULTS}

Crystal patch formation. The formation of the crystal order can be analyzed through the bond-orientational order parameter $\psi_{6}^{j}=\frac{1}{Z_{j}} \sum_{k} \exp \left(6 i \theta_{j k}\right)$ for each particle $j$, where the sum runs over the $Z_{j}$ nearest neighbors of particle $j$, and $\theta_{j k}$ is the angle between the $j-k$ bond and a fixed direction. This order parameter takes a value $\psi_{6}^{j}=0$ for a liquid particle, and $\psi_{6}^{j}=1$ for a crystal particle.

Sequences 2(a) and 2(b) show the early crystallization process from an initial liquid, on two differently curved substrates [in Fig. 2(a) $K_{\max } a^{2}=0.64$, and in Fig. 2(b) $K_{\max } a^{2}=1.18$, with $a$ being the lattice constant]. To better visualize the crystal regions, here we have superimposed the bond order-parameter maps $\psi_{6}$ with the particles. Note that in both cases the formation of hexagonal order (red regions) starts in the flatter regions of the substrate. In flat systems the initial crystal seeds of a liquid-solid transition are randomly distributed throughout the system $[1,5]$. On the contrary, here the curvature breaks the isotropy of space, and the formation of hexagonal patches is favored on regions where the Gaussian curvature is small. This is a consequence of the geometrical frustration created by the curvature, where the geometry produces distortions that increase the strain energy in the lattice, inhibiting the formation of perfectly hexagonal patches in regions of high curvature.

This phenomenon can be clearly observed by studying the distribution of the crystal order parameter $\psi_{6}$ as a function of the local value of the Gaussian curvature $K$. Figures 3(a) and 3(b) show $\psi_{6}$ for early and long times during the 
(a)

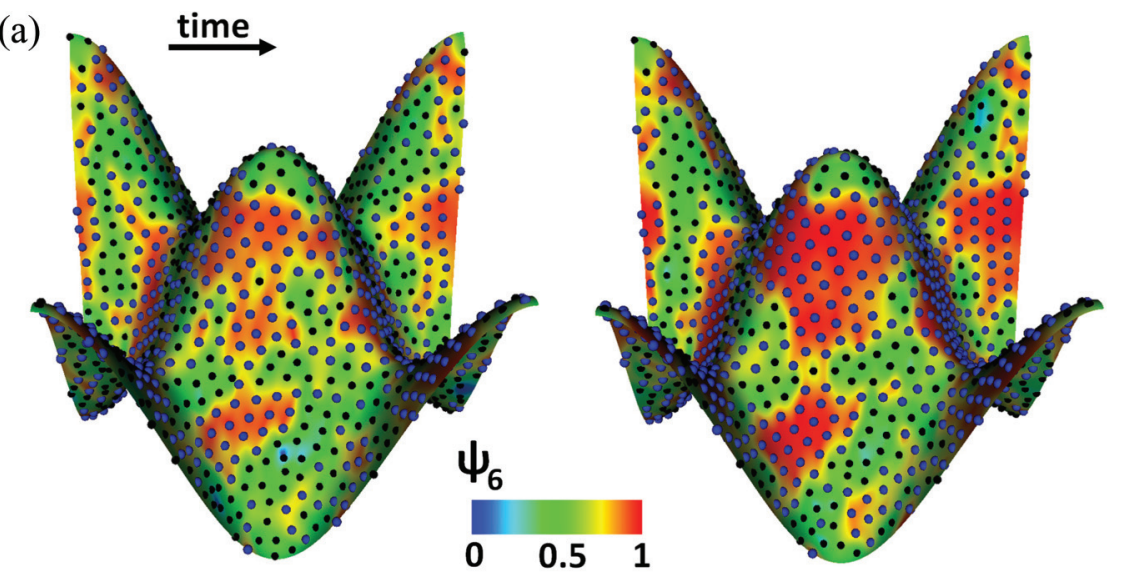

(b)
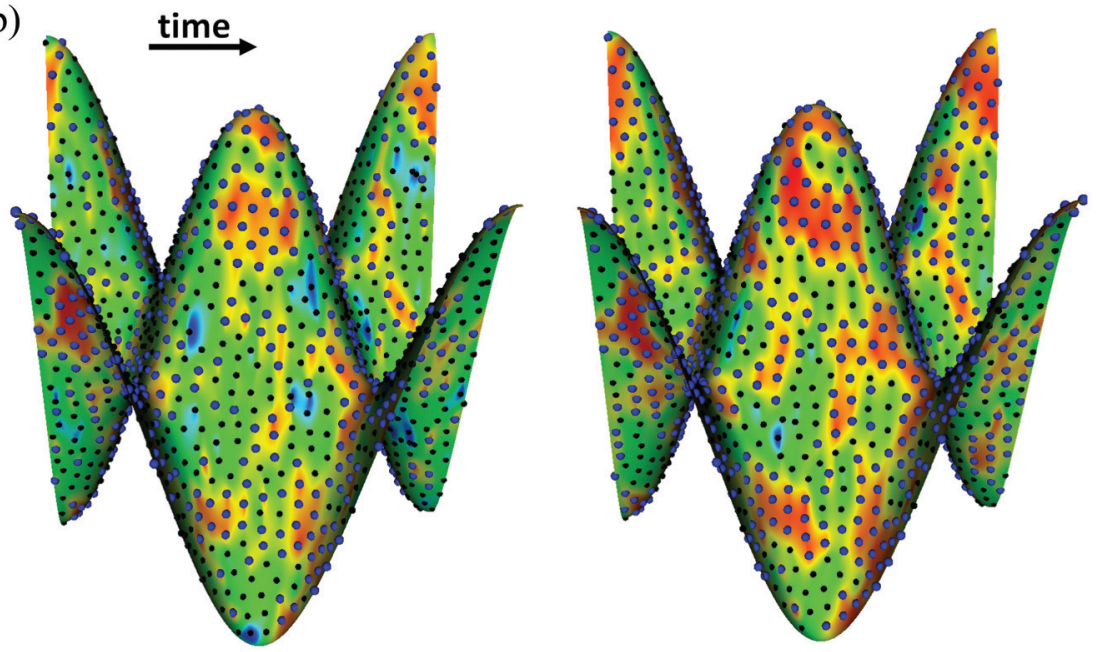
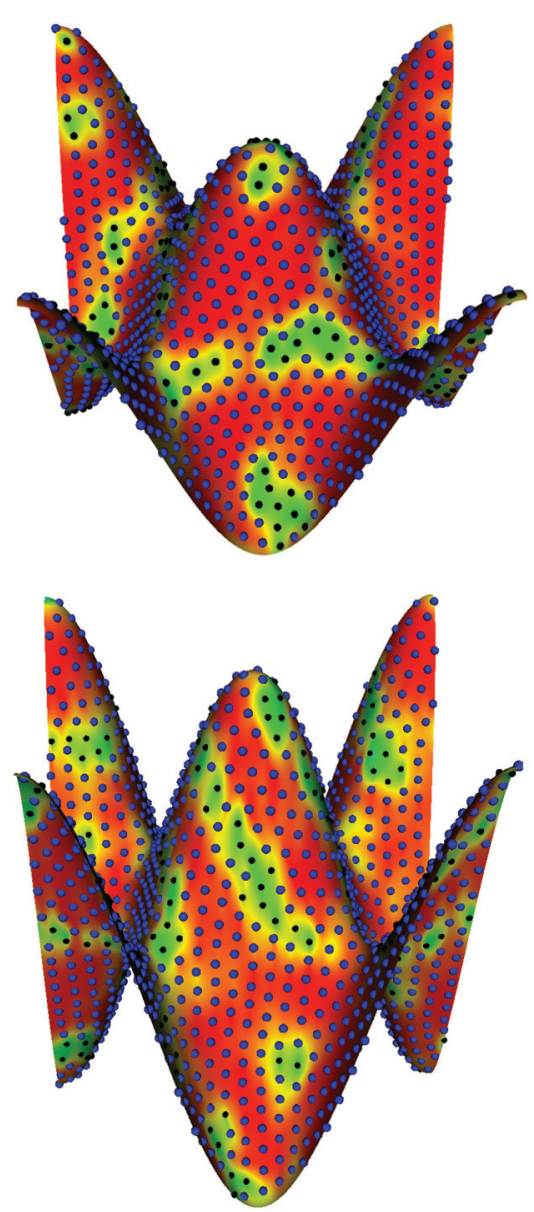

FIG. 2. (Color online) Formation of crystal domains from an initial liquid phase at curvatures $K_{\max } a^{2}=0.64$ (a) and $K_{\max } a^{2}=1.18$ (b). Horizontal panels correspond to the same evolution times $[t=1500$ (top), $t=3000$ (middle), and $t=15000$ (bottom)]. While crystal particles are drawn as big blue (gray) spheres, liquidlike particles are drawn as smaller black spheres. Note the formation of the early hexagonal nuclei in the flattest regions of the substrate (left and middle panels). For large curvatures the hexagonal domains start to develop rather elongated shapes [compare the red (dark gray) regions in the right panels of (a) and (b)].

crystallization on substrates of increasing curvature. Since the geometric frustration is reduced by disclinations that locally reduce crystallinity, by increasing the maximum curvature $K_{\max }$ the crystallinity becomes sharply peaked around the flatter regions $(K \sim 0)$ at early and long times. Note that as the curvature of the system is increased, the confinement of crystal seeds to regions of low curvature is stronger.

The global process of crystallization can be tracked through the fraction of particles involved in crystals, $f_{C}=$ $\sum_{j} \psi_{6}^{j} / \sum_{j}$. Figure 3(c) shows the temporal evolution of $f_{C}$. This plot shows that the dynamics become slower and the fraction of crystallized particles decreases by increasing the curvature. Thus the curvature not only frustrates the formation of hexagonal domains in regions of high curvature, but also affects the dynamics towards the equilibrium state.

Defect structures. We observed that the early crystalline seeds grow through the addition of new hexagonally packed particles, invading the rest of the substrate. However, the growth is not homogeneous and the crystals grow preferentially faster along regions of low curvature. At intermediate time scales, where the system remains far from equilibrium, we observed defect configurations similar to those observed in curved colloidal crystals (Fig. 4). Here the different structures of defects that participate in the dynamics of crystallization are dislocations, pleats (linear arrays of dislocations with variable distance between them [14]), free disclinations, and scars (linear arrays of dislocations and disclinations with a net topological charge) [9]. During crystallization in flat space, the domain structure is dominated by triple points (regions where three grains meet) [32], [33]; isolated disclinations are absent [34-36]. However, due to curvature, here the triple points become unstable and the mechanism of domain growth produces scars and pleats (see Fig. 4) decorating the interface between grains having a large orientational mismatch, while free disclinations are rapidly stabilized in the highly curved regions in order to screen the geometric potential [27].

Pair correlations. The relaxation towards the ordered crystal requires the annihilation of the excess defects, while keeping the total topological charge equal to zero. A standard tool to study the ordering process is the pair-correlation function $g(r)$, giving the probability of finding a particle at distance $r$ away from a reference particle [37] (see Appendix C). For a perfectly ordered crystal, $g(r)$ is a periodic array of $\delta$ functions whose positions and heights are determined by the lattice 

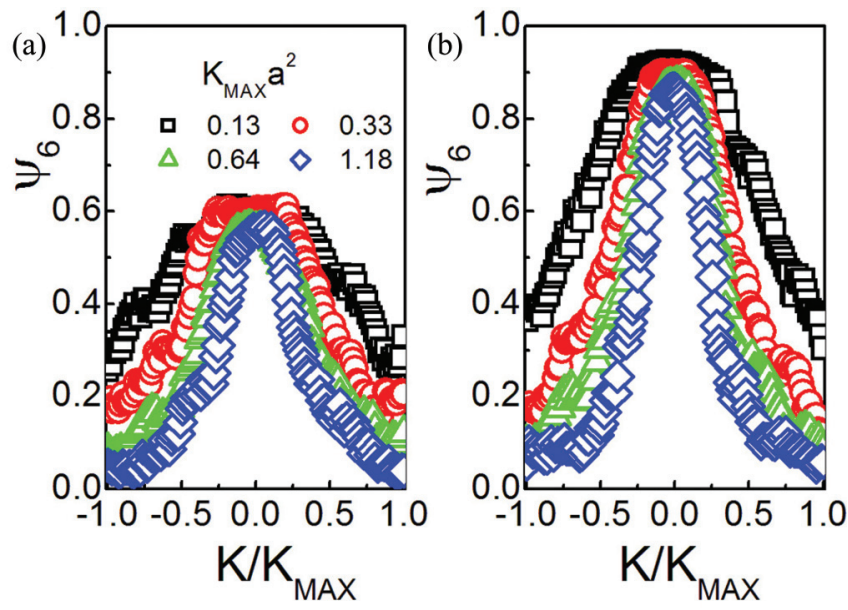

(c)

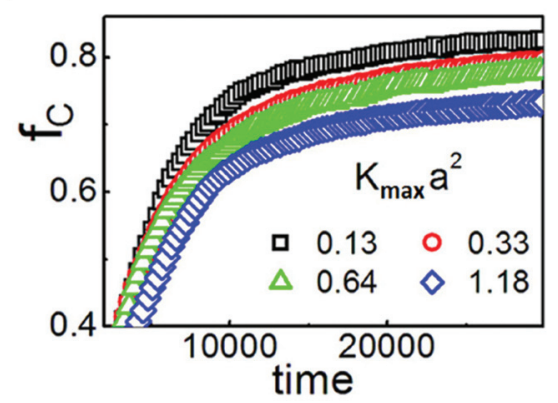

FIG. 3. (Color online) Distribution of the order parameter $\psi_{6}$ as a function of the Gaussian curvature $K$ at times $t=3000$ (a) and $t=20000$ (b). The curves correspond to different substrates (curvatures indicated in the figure). (c) Temporal evolution of the crystal fraction $f_{C}$. Here $a$ is the lattice constant.

order. Since disorder strongly affects the peak structure, it has been previously found that $g(r)$ can be employed to study the degree of ordering. Figure 5 shows $g(r)$ for a highly curved substrate $\left(K_{\max } a^{2} \sim 1\right)$ at early and long times. The process of ordering is evidenced by the sharpening of higher-order peaks in $g(r)$, which correspond to the peak structure found for a hexagonal lattice (peaks located at $r / a=1, \sqrt{3}, 2, \sqrt{7}, 3, \ldots$ ). When comparing $g(r)$ at a fixed time, we observe that the amplitude of the first peak in $g(r)$ systematically decreases upon increasing the maximum curvature $K_{\max }$. However, unlike other systems where the degree of order affects the distribution of peak heights, here we observe that the ratio between peak heights is relatively insensitive to the curvature (inset of Fig. 5). Thus, although at high curvatures the system contains a number of isolated disclinations and other defect structures, their highly energetic elastic distortions are deeply relaxed by the geometry.

Defect evolution and correlation length. The coarsening dynamics can also be elucidated by tracking the motion and distribution of topological defects. At early times, the disorder in the lattice screens out the local curvature, producing a roughly random distribution of defects throughout the system [38]. At this early stage the dynamics are relatively unaffected by the curvature and thus the process of defect annihilation can be expected to follow "line-tension-driven"Allen-Cahn dynamics [32,39]. As time proceeds, most defects condense along domain walls in pleats and scars, while their dynamics

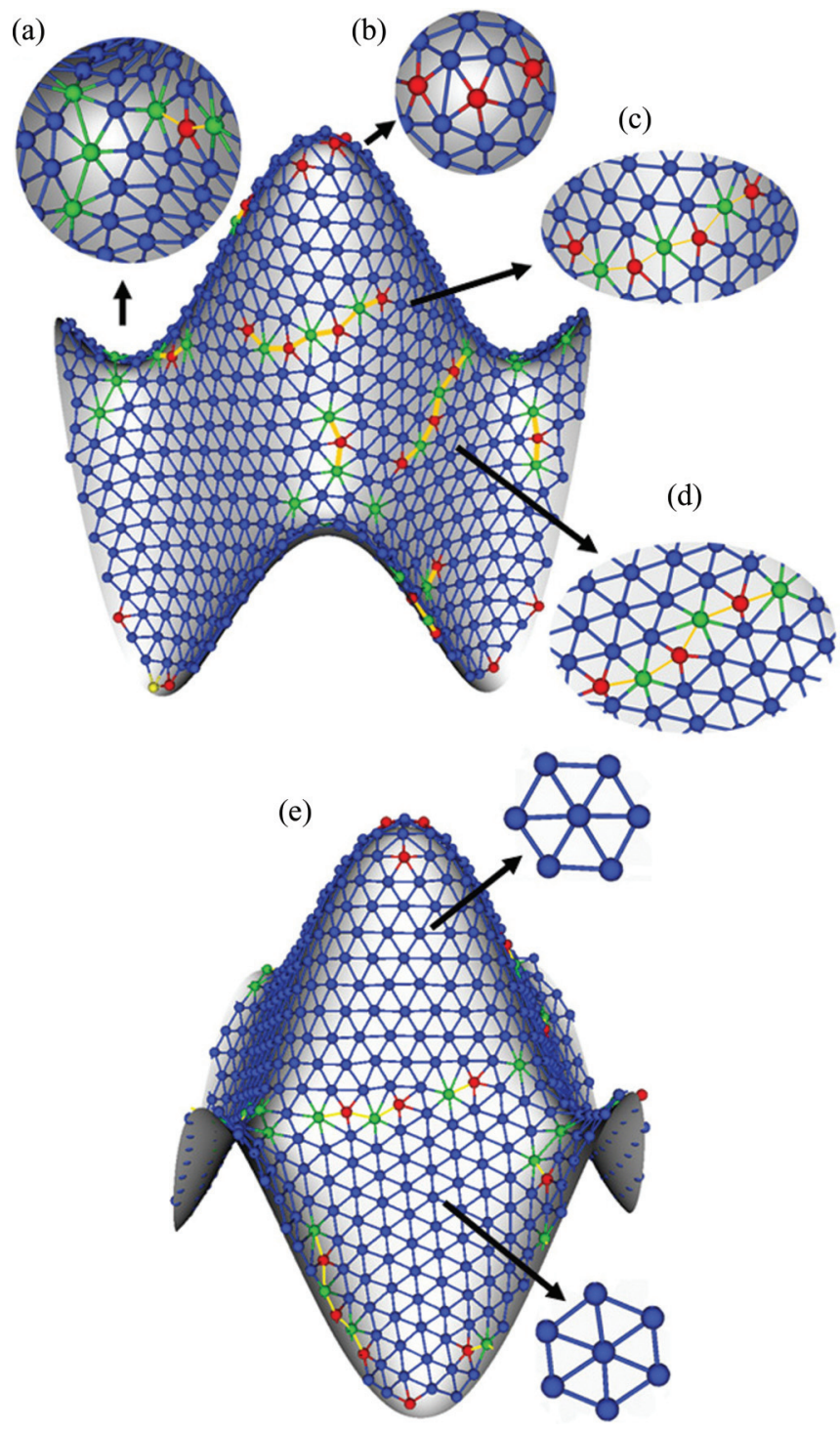

FIG. 4. (Color online) Negative [panel (a)] and positive [panel (b)] disclinations located at saddles and bumps, respectively, reduce the geometric frustration produced in regions with high curvature. Scars (c) and pleats (d) delimit different curved hexagonal crystals. (e) Domain structure observed at long times. Here grain boundaries become pinned to regions of low curvature, slowing the kinetics of ordering.

of diffusion and annihilation are deeply influenced by both isolated disclinations $[40,41]$ and curvature. The sequence of Fig. 6(a) shows the mechanism of ordering most often observed in regions of high curvature. Here the partially screened strain field of the disclinations located at the bumps acts as a sink for linear arrays of defects that diffuse and annihilate at these highly curved regions.

The analysis of the defect structures and the annihilation mechanism reveals that in addition to the local traps produced by regions of positive (negative) curvature for the motion of positive (negative) disclinations, a slow glassylike ordering results from the pinning of linear arrays of defects to regions of low curvature. Figure 4(e) shows a typical pinning of a domain wall connecting negative disclinations, located at saddle points. We found that the deep traps produced by 


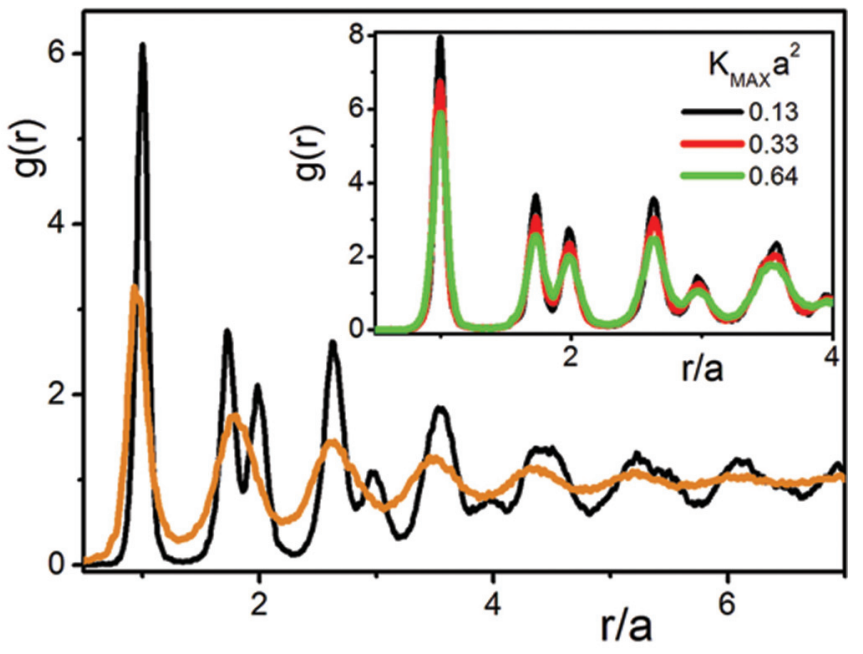

FIG. 5. (Color online) Pair correlations $g(r)$ at short and long times shown as orange (gray) and black lines for a highly curved substrate $\left(K_{\max } a^{2}=1.18\right)$. Inset: $g(r)$ for systems of increasing curvature $\left(t=3 \times 10^{5}\right)$.

the regions of low curvature generate very stable domain structures that dramatically slow down dynamics by freezing the ordering kinetics [42-44]. Note, however, that this slowing down is not produced by the formation of a glassy phase, but rather is related to an arrested state, which is unable to reach equilibrium. Although in Euclidean space the slow dynamics

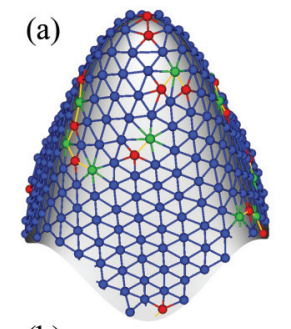

(b)

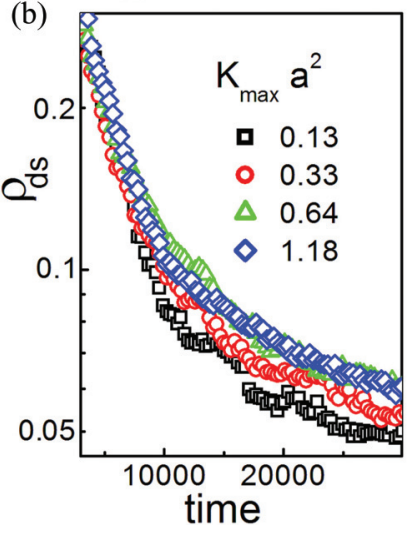

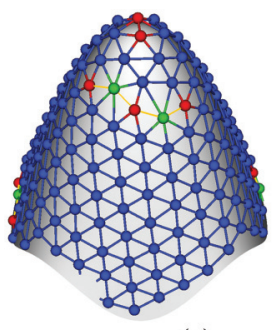

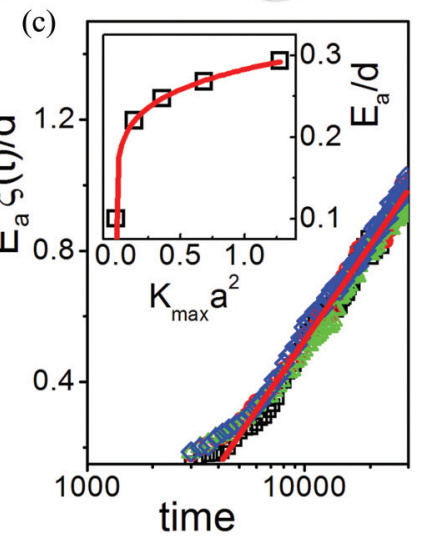

FIG. 6. (Color online) (a) Linear arrays of defects decorating domain walls diffuse to regions of high curvature where they are absorbed by isolated disclinations. Panels (b) and (c) show $\rho_{d s}$ and $\xi$ as functions of time, for systems with different underlying curvatures. Note that upon normalization by $E_{a}, \xi$ asymptotically follows a similar slow logarithmic evolution, irrespective of $K_{\max }$. The inset of panel (c) shows $E_{a}$ as a function of $K_{\max }$. The value of $E_{a}$ found in flat systems $\left(K_{\max }=0\right)$ has also been included. The line represents a power-law fit, $E_{a} \sim K_{\max }^{0.23}$. of systems with competing interactions has for years been related to the formation of glasses, recent results indicate that the dynamics in the two cases would be intrinsically different $[42,45]$.

This slowing down in the dynamics can be tracked through the time evolution of the areal density of dislocations $\rho_{d s}$ [Fig. 6(b)]. It can be observed that, in agreement with the results shown in Fig. 3(c), at short times the dynamics are relatively insensitive to the curvature, while a clear coupling with the geometry appears at intermediate and long times. In this regime, upon increasing the maximum curvature, the rate of defect annihilation decreases and larger contents of defects are involved in the relaxation process.

Since defect structures mainly decorate domain walls, a characteristic length scale $\xi$, defined in terms of $\rho_{d s}$ as $\xi \sim d / \rho_{d s}$, provides a measure of the average domain size [32-35]. Here $d \sim 2 a$ is the average distance between dislocations along a domain wall. In coarsening systems where a free-energy barrier $U_{a}$ is involved in the growth of the domains, the rate of change of the correlation length takes the form $\frac{d \xi}{d t}=\exp \left(-\frac{U_{a}}{k T}\right)$ [32]. Since here the energy excess is produced mainly by the domain walls, we have $U_{a} / k T=$ $E_{a} \xi / d$, where $E_{a}$ is a free-energy parameter characterizing the strength of the barrier. Solving for $\xi$, the asymptotic behavior of $\xi$ becomes $\xi(t) \sim \frac{d}{E_{a}} \ln \frac{E_{a}}{d} t$. Figure 6(c) shows the temporal evolution of $E_{a} \xi(t) / d$ for substrates with different curvature $K_{\max }$. In agreement with an activated mechanism, here we find that, in the long-time regime, $\xi$ is consistent with a logarithmic dependence on time. We also found that the mean-square displacement of the particles is consistent with a logarithmic behavior. The inset of Fig. 6(c) also shows the dependence of $E_{a}$ as a function of the maximum curvature of the substrate $K_{\max }$. The activation energy for a flat system has also been included for comparison. In agreement with the qualitative observations, the activation energy grows continuously with the maximum curvature, indicating that the pinning of defects becomes stronger for larger curvatures. However, the dependence on curvature is relatively weak, with $E_{a}$ following a power law $E_{a} \sim K_{\max }^{\eta}$ with a relatively small exponent $(\eta=0.23)$.

\section{CONCLUSIONS}

In this work we have studied how the mechanisms of formation of a two-dimensional crystal phase are modified by the presence of curvature. We observed that order is triggered earlier on the flattest regions of the substrate, where the frustration in the lattice due to the curvature is smaller. The frustration to form the crystal induced by the geometry of the substrate can be partially reduced by different topological defect structures.

Curved polycrystals show ordering mechanisms somewhat similar to those of their flat counterparts, modified by curvature. As the coupling between varying curvature and crystal order induces the pinning of grain boundaries, glassylike dynamics arise which may completely inhibit the appearance of the ground-state structure. These dynamic effects should be taken into account not only at the onset of a phase transition during crystallization or melting, but also in the design of applications that require well-ordered self-assembled 
structures, like surfaces functionalized with ordered arrays of topological defects.

\section{ACKNOWLEDGMENTS}

This work was supported by the National Research Council of Argentina, CONICET, ANPCyT, Universidad Nacional del Sur and the National Science Foundation MRSEC Program through the Princeton Center for Complex Materials (Grant No. DMR-0819860).

\section{APPENDIX A: EVOLUTION EQUATION}

By combining the free-energy functional [Eq. (1)] with the relaxational equation [Eq. (2)], we obtain a partial differential equation describing the evolution of the order parameter during crystallization:

$$
\frac{\partial \psi}{\partial t}=M \nabla_{\mathrm{LB}}^{2}\left[f(\psi)-D \nabla_{\mathrm{LB}}^{2} \psi\right]-M b \psi,
$$

where $f(\psi)=d W(\psi) / d \psi=-\gamma \psi+v \psi^{2}+u \psi^{3}$, and $\nabla_{\mathrm{LB}}^{2}$ is the Laplace-Beltrami operator which in its general form can be written as [46]

$$
\nabla_{\mathrm{LB}}^{2} \equiv \frac{1}{\sqrt{g}} \frac{\partial}{\partial x^{i}}\left(g^{i j} \sqrt{g} \frac{\partial}{\partial x^{j}}\right) .
$$

Here $g$ is the determinant of the metric of the substrate $g_{i j}=$ $\frac{\partial \mathbf{R}}{\partial x^{i}} \cdot \frac{\partial \mathbf{R}}{\partial x^{j}}$, and $g_{i k} g^{k j}=\delta_{i}^{j}$.

In the present work the evolution equation for the order parameter is numerically solved on the sinusoidal geometry:

$$
\mathbf{R}\left(x^{1}, x^{2}\right)=x^{1} \mathbf{i}+x^{2} \mathbf{j}+A \cos \left(2 \pi x^{1} / L\right) \cos \left(2 \pi x^{2} / L\right) \mathbf{k} .
$$

We have implemented a finite difference algorithm, centered in space and forward in time, on a $1024 \times 1024$ square grid with periodic boundary conditions. In order to avoid any numerical coupling with the underlying lattice, the parameters of the free energy and the number of lattice points of the numerical grid are chosen such that the particle diameter is represented by at least 10 lattice points. The initial liquid phase is modeled by random fluctuations in the order parameter $\psi$. The evolution of particles during crystallization is followed by the identification and tracking of the maxima of the order parameter [47].

\section{APPENDIX B: DELAUNAY TRIANGULATION ON ARBITRARILY CURVED SURFACES}

As pointed out above, the dominant features of the system can be analyzed by the use of Delaunay triangulation [35]. Through the triangulation, it is possible to obtain structural parameters such as the particle's first neighbors, degree of crystallinity, or topological defects [32-35]. Once the positions of the particles are determined, the analysis starts by calculating each particle's first neighbors. To do that, we implemented the Fast Marching algorithm [48]. Here a propagating front starting from each particle is obtained by solving the evolution equation [Figs. 7(a) and 7(b)],

$$
\frac{\partial \Lambda}{\partial t}+\vec{u} \cdot \vec{\nabla} \Lambda=0
$$

where $\vec{u}$ is the propagation velocity $(|\vec{u}|=1)$. The resulting function $\Lambda$ gives the distance to the initial point, such that the
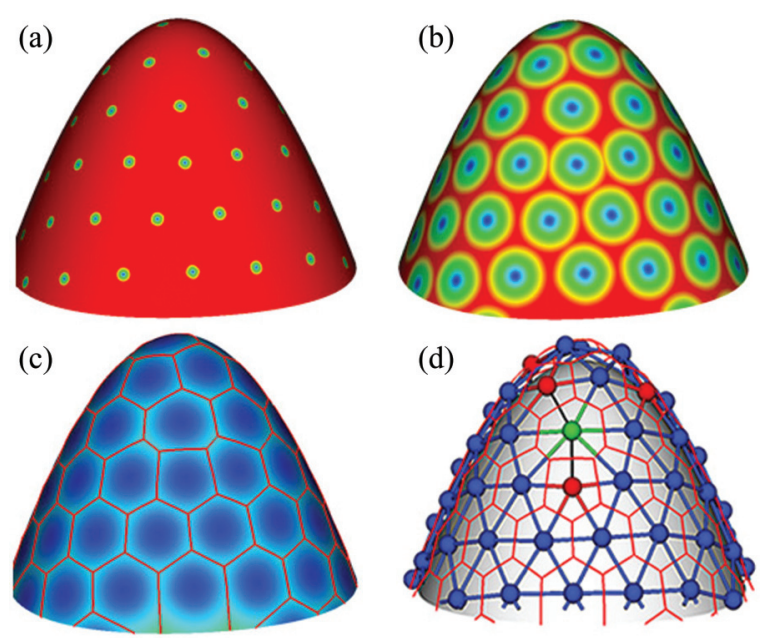

FIG. 7. (Color online) Delaunay triangulation construction on curved surfaces by the use of a fast front marching technique. The propagation of fronts from each particle [(a) and (b)] allows the determination of the first neighbors, Voronoi diagram (c), and Delaunay triangulation and topological defects (d).

geodesic distance between two arbitrary points on the surface is easily determined.

With the geodesic distances between particles we construct the Voronoi diagrams and Delaunay triangulations and also locate the particles' first neighbors and determine their coordination numbers $Z$ [Figs. 7(c) and 7(d)]. This allows the identification of topological defects in the structure.

(a)
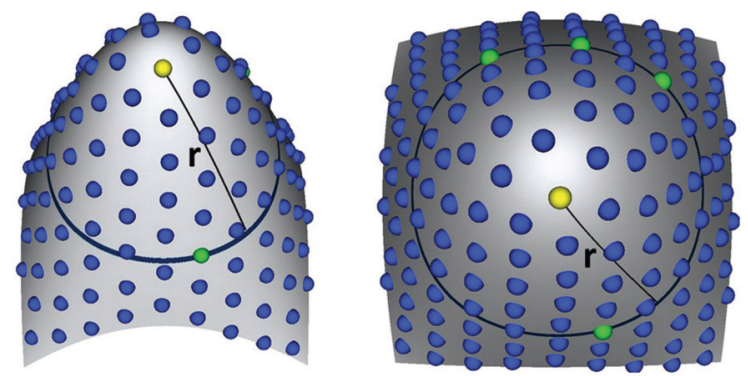

(b)

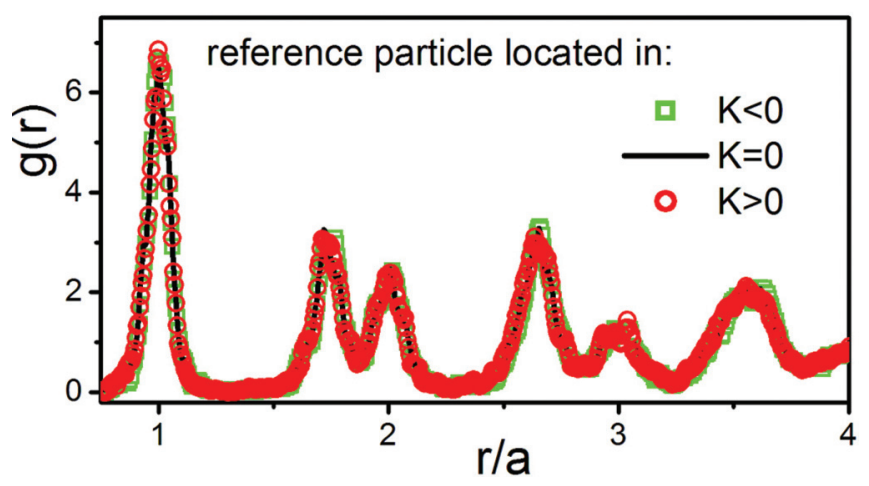

FIG. 8. (Color online) (a) Scheme showing the calculation of pair correlations in curved space. Here the reference particle is in yellow (light gray). (b) Pair correlations of a crystal-like structure calculated using particles located in regions of negative, null, and positive curvatures. 


\section{APPENDIX C: PAIR CORRELATIONS}

In curved space, the pair-correlation function $g(r)$ can be generalized as the average number of particles with geodesic distances between $r$ and $r+d r$ [37]. Figure 8(a) shows a scheme of the calculation of $g(r)$ through the use of geodesic circles. Here the spatial correlations between the particle's locations produce peaks in $g(r)$ at characteristic distances $r^{*}$ related to the lattice structure [Fig. 8(b)].

Contrary to $3 \mathrm{D}$ or flat $2 \mathrm{D}$ systems, or to homogeneously curved systems (spheres or pseudospheres), on sinusoidal substrates one should question whether it is proper to simply average $g(r)$ calculated for different particles, because the varying curvature breaks the homogeneity of space. However, as shown in Fig. 8(b), we have only found small differences in $g(r)$ when calculated with reference particles located in curved or flat regions. Thus the average of $g(r)$ for the different particles represents a good measure of the state of order of the system. The regularity in the lattice structure, producing similar pair correlations independent of the reference particle's location, comes from the ability to pack topological defects in the most curved regions of the substrate.
[1] P. M. Chaikin and T. C. Lubensky, Principles of Condensed Matter Physics (Cambridge University Press, Cambridge, UK, 1995).

[2] S. Alexander and J. McTague, Phys. Rev. Lett. 41, 702 (1978).

[3] U. Gasser, E. R. Weeks, A. Schofield, P. N. Pusey, and D. A. Weitz, Science 292, 258 (2001).

[4] L. R. Gómez and D. A. Vega, Phys. Rev. E 83, 021501 (2011).

[5] D. A. Vega and L. R. Gómez, Phys. Rev. E 79, 051607 (2009).

[6] W. F. Harris, in Fundamental Aspects of Dislocation Theory, Natl. Bur. Stand. (US) Spec. Publ. No. 317, edited by J. A. Simmons, R. de Wit, and R. Bullough, Vol. I (US GPO, Washington, DC, 1970), p. 579.

[7] F. R. N. Navarro, in Fundamental Aspects of Dislocation Theory, Natl. Bur. Stand. (US) Spec. Publ. No. 317, edited by J. A. Simmons, R. de Wit, and R. Bullough, Vol. I (US GPO, Washington, DC, 1970), p. 593.

[8] A. Pérez-Garrido, M. J. W. Dodgson, and M. A. Moore, Phys. Rev. B 56, 3640 (1997).

[9] A. R. Bausch, M. J. Bowick, A. Cacciuto, A. D. Dinsmore, M. F. Hsu, D. R. Nelson, M. G. Nikolaides, A. Travesset, and D. A. Weitz, Science 299, 1716 (2003).

[10] C. Li, X. Zhang, and Z. Cao, Science 309, 909 (2005).

[11] V. Vitelli, J. B. Lucks, and D. R. Nelson, Proc. Natl. Acad. Sci. USA 103, 12323 (2006).

[12] A. Hexemer, V. Vitelli, E. J. Kramer, and G. H. Fredrickson, Phys. Rev. E 76, 051604 (2007).

[13] M. J. Bowick and L. Giomi, Adv. Phys. 58, 449 (2009).

[14] W. T. M. Irvine, V. Vitelli, and P. M. Chaikin, Nature (London) 468, 947 (2010).

[15] Z. Yao and M. Olvera de la Cruz, Phys. Rev. E 87, 012603 (2013).

[16] W. T. M. Irvine and V. Vitelli, Soft Matter 8, 10123 (2012).

[17] P. Lipowsky, M. J. Bowick, J. H. Meinke, D. R. Nelson, and A. R. Bausch, Nat. Mater. 4, 407 (2005).

[18] W. T. M. Irvine, M. Bowick, and P. M. Chaikin, Nat. Mater. 11, 948 (2012).

[19] A. Hexemer, Ph.D. thesis, University of California, Santa Barbara, 2006.

[20] C. D. Santangelo, V. Vitelli, R. D. Kamien, and D. R. Nelson, Phys. Rev. Lett. 99, 017801 (2007).

[21] R. D. Kamien, D. R. Nelson, C. D. Santangelo, and V. Vitelli, Phys. Rev. E 80, 051703 (2009).

[22] L. R. Gómez and D. A. Vega, Phys. Rev. E 79, 031701 (2009).
[23] I. Hasegawa and H. Shima, Mod. Phys. Lett. B 25, 581 (2011).

[24] T. Lopez-Leon, V. Koning, K. B. S. Devaiah, V. Vitelli, and A. Fernandez-Nieves, Nature Phys. 7, 391 (2011).

[25] G. Napoli and L. Vergori, Phys. Rev. Lett. 108, 207803 (2012).

[26] N. Xie, W. Li, F. Qiu, and A.-C. Shi, Soft Matter 9, 536 (2013).

[27] D. R. Nelson, Nano Lett. 2, 1125 (2002).

[28] G. A. DeVries, M. Brunnbauer, Y. Hu, A. M. Jackson, B. Long, B. T. Neltner, O. Uzun, B. H. Wunsch, and F. Stellacci, Science 315, 358 (2007).

[29] T. Ohta and K. Kawasaki, Macromolecules 19, 2621 (1986).

[30] M. Seul and D. Andelman, Science 267, 476 (1995).

[31] B. O’Neill, Elementary Differential Geometry (Academic, New York, 1997).

[32] L. R. Gómez, E. M. Vallés, and D. A. Vega, Phys. Rev. Lett. 97, 188302 (2006).

[33] A. D. Pezzutti, L. R. Gómez, M. A. Villar, and D. A. Vega, Europhys. Lett. 87, 66003 (2009).

[34] D. A. Vega, C. K. Harrison, D. E. Angelescu, M. L. Trawick, D. A. Huse, P. M. Chaikin, and R. A. Register, Phys. Rev. E 71, 061803 (2005).

[35] C. K. Harrison, D. E. Angelescu, M. Trawick, Z. Cheng, D. A. Huse, P. M. Chaikin, D. A. Vega, J. M. Sebastian, R. A. Register, and D. H. Adamson, Europhys. Lett. 67, 800 (2004).

[36] C. K. Harrison, Z. Cheng, S. Sethuraman, D. A. Huse, P. M. Chaikin, D. A. Vega, J. M. Sebastian, R. A. Register, and D. H. Adamson, Phys. Rev. E 66, 011706 (2002).

[37] M. Rubinstein and D. R. Nelson, Phys. Rev. B 28, 6377 (1983).

[38] G. Tarjus, F. Sausset, and P. Viot, Adv. Chem. Phys. 148, 251 (2012).

[39] S. M. Allen and J. W. Cahn, Acta Metall. 27, 1058 (1979).

[40] R. deWit, J. Appl. Phys. 42, 3304 (1971).

[41] W. F. Harris and L. E. Scriven, J. Appl. Phys. 42, 3309 (1971).

[42] L. Berthier and J. Biroli, Rev. Mod. Phys. 83, 587 (2011).

[43] I. M. Lifshitz, Sov. Phys. JETP 15, 939 (1962).

[44] S. A. Safran, Phys. Rev. Lett. 46, 1581 (1981).

[45] P. L. Geissler and D. R. Reichman, Phys. Rev. E 69, 021501 (2004).

[46] D. J. Struik, Lectures on Classical Differential Geometry (Dover, New York, 1988).

[47] J. C. Crocker and D. G. Grier, J. Colloid Interface Sci. 179, 298 (1996).

[48] J. A. Sethian and P. Smereka, Annu. Rev. Fluid Mech. 35, 341 (2003). 